\title{
Spatial memory in the real world: long-term representations of everyday environments
}

\author{
Steven A. Marchette • Ashok Yerramsetti • \\ Thomas J. Burns • Amy L. Shelton
}

Published online: 17 May 2011

(C) Psychonomic Society, Inc. 2011

\begin{abstract}
When people learn an environment, they appear to establish a principle orientation just as they would determine the "top" of a novel object. Evidence for reference orientations has largely come from observations of orientation dependence in pointing judgments: Participants are most accurate when asked to recall the space from a particular orientation. However, these investigations have used highly constrained encoding in both time-scale and navigational goals, leaving open the possibility that larger spaces experienced during navigational learning depend on a different organizational scheme. To test this possibility, we asked undergraduates to perform judgments of relative direction on familiar landmarks around their well-learned campus. Participants showed clear evidence for a single reference orientation, generally aligned along salient axes defined by the buildings and paths. This result argues that representing space involves the establishment of a reference orientation, a requirement that endures over repeated exposures and extensive experience.
\end{abstract}

\section{Keywords Spatial cognition · Memory $\cdot$ Memory}

organization $\cdot$ Representation

Imagine you are skimming this article quickly on your way to journal club. Despite directing your attention to reading, you readily make your way down the hall, perhaps detouring around a recent renovation project, and arrive safely at the seminar room ready to discuss. In addition to revealing your savvy time management skills, you would have demonstrated the fluent spatial memory abilities that

S. A. Marchette $\cdot$ A. Yerramsetti $\cdot$ T. J. Burns $\cdot$ A. L. Shelton $(\triangle)$ Dept. of Psych \& Brain Sciences, Johns Hopkins University,

3400 N Charles St,

Baltimore, MD 21218, USA

e-mail: ashelton@jhu.edu underlie many of our daily activities. Humans have the capacity to quickly plan and execute routes through complex environments by accessing spatial memory representations. Given the wealth of navigationally relevant information that must be represented (object locations, environmental structure), successful encoding and subsequent retrieval depend heavily on how the memory representation is organized. As a result, the organizational principles that guide these representations have received considerable attention in the spatial memory literature. In the present article, we consider one of the most consistently observed and supported principles: the establishment of a principal reference orientation (for a review, see McNamara \& Valiquette, 2004; Shelton \& Yamamoto, 2009).

Principal Reference Theory (PRT) has emerged as a theoretical framework to explain orientation-dependent memory performance. When people are asked to imagine different perspectives of a learned space, they tend to be fastest and/or most accurate for one orientation relative to all others (e.g., Greenauer \& Waller, 2008, 2010; Hintzman, O’Dell \& Arndt, 1981; Kelly, Avraamides \& Loomis, 2007; Kelly \& McNamara, 2008; Marchette \& Shelton, 2010; May, 2007; Mou \& McNamara, 2002; Mou, Liu \& McNamara, 2009; Mou, Zhao \& McNamara, 2007; Roskos-Ewoldsen, McNamara, Shelton \& Carr, 1998; Shelton \& Marchette, 2010; Shelton \& McNamara 1997, 2001a, 2004a, b; Valiquette \& McNamara, 2007; Yamamoto \& Shelton 2005, 2007, 2009). According to PRT, representing space involves selecting a principle orientation as the reference for encoding locations (e.g., Shelton \& McNamara, 2001a; Werner \& Schmidt, 1999). Over the last decade, mounting evidence has suggested that reference orientation selection is based on a hierarchy of cues. Egocentric experience appears to provide a default organization for memory; in the absence of other organizational cues, an 
egocentrically experienced orientation will serve as the reference (Greenauer \& Waller, 2008; Shelton \& McNamara, 1997; 2001a, b). Salient organizational cues are a common feature in natural environments, however, and these cues can adjudicate when a space has been experienced from more than one orientation (McNamara, Rump \& Werner, 2003; Shelton \& McNamara, 2001a; Werner \& Schmidt, 1999). For example, the walls of a room provide a strong environmental frame, and an orientation aligned with the walls will be selected over an orientation misaligned with the walls (Shelton \& McNamara, 2001a, Exp.3). In addition, strong organizational cues can even drive selection of a reference orientation that was never experienced during learning, including strong columnar organization of locations (e.g. Mou \& McNamara, 2002), an axis of bilateral symmetry (e.g., Mou et al., 2007), or aligning all of the object axes with a single orientation in the space (Marchette \& Shelton, 2010). Taken together, this body of evidence suggests that the selection of a reference orientation involves the opportunistic use of available reference frames, with interactions between egocentric experience and environmental cues.

Although these studies have provided a strong foundation for thinking about the organizational principles of spatial memory, the conditions of these experimental settings differ dramatically from the conditions involved in our daily interactions with space on many critical features. First, studies have frequently used room-sized (e.g., Greenauer \& Waller, 2010; Mou et al., 2007; Shelton \& McNamara, 2001a) or table-top (e.g., RoskosEwoldsen et al., 1998; Shelton \& McNamara, 2001b, 2004b) displays in order to tightly control exposure to specific orientations and limit movement in the space. In such situations, the whole display can be viewed simultaneously with small movements (i.e., vista scale), whereas many spaces in which we navigate daily have discrete locations that are not simultaneously in view (i.e., environmental scale). It is possible that principles governing the organization within a vista-scale space differ from those that govern integration across subsequent local vistas in an environmental-scale space (e.g., Acredolo, 1981; Montello, 1993; Presson, DeLange \& Hazelrigg, 1989). A small number of studies have examined memory for large-scale real environments (McNamara et al., 2003) or virtual spaces (e.g., Shelton \& McNamara, 2004a) and have shown the characteristic preferred orientation, despite the need to traverse multiple path legs. As such, differences in scale do not appear to be a major factor affecting these broad organizational principles on its own.

More critically, however, most studies differ from familiar spaces at a more fundamental experiential level: Participants learn a layout in a limited number of exposures to the environment - typically, one - and with a limited set of learning goals. Although learning may be "broken up" into mini-sessions between which participants close their eyes, the entire experience with the space takes place in the context of a single experimental session. In addition, the entire encoding experience with the environment lasts around 20-30 min with retrieval following shortly after (e.g., Greenauer \& Waller, 2008, 2010; Marchette \& Shelton, 2010; McNamara et al., 2003; Mou et al., 2009; Mou \& McNamara, 2002; Mou et al., 2007; Shelton \& McNamara, 2001a, b; Valiquette \& McNamara, 2007; Yamamoto \& Shelton 2005, 2007, 2009). Most familiar spaces are learned over extended time periods-days, weeks, months, years-with navigational goals varying with different exposures. In the present article, we consider how this variable exposure might affect organizational strategies/principles.

It is possible that the experimental cases offer insufficient experience to extend the representation to a more generalized representation. Studies showing a single preferred orientation after exposure to multiple views argue against the strongest version of this limitation argument (e.g., Kelly \& McNamara, 2010; Shelton \& McNamara, 1997; 2001a; Yamamoto \& Shelton, 2005); however, these studies still use highly constrained encoding in terms of both time and navigational goals. As such, it is possible that the observed orientation dependence reflects either a temporary strategy that works well for short-term retention or a "starter" representation on which to build. For the latter, it is possible that over many different exposures with variable navigational goals, one can establish a richer, more flexible, and essentially orientation-independent representation. ${ }^{1}$

A small number of studies have examined large-scale familiar spaces learned under more natural conditions (e.g., Anooshian \& Young, 1981; Bryant, 1982, 1984; Buttenfield, 1986; Hirtle \& Jonides, 1985; Kitchin \& Fortheringham, 1997; Montello, 1991; Rovine \& Weisman, 1989; Sholl, 1987; Tlauka, 2006; Werner \& Schmidt, 1999), but these have provided only limited information with respect to reference frame selection. For example, Sholl demonstrated that participants were faster and more accurate at locating local landmarks when facing a more frequently experienced direction. In a converging line of evidence, Tlauka found that landmark locations in a large-scale environment were more readily recalled from some imagined locations than from others. Montello (1991) showed that pedestrians were more accurate at locating local landmarks when traveling on a

\footnotetext{
${ }^{1}$ One could engage in the philosophical argument of multiple representations versus a single flexible representation. For the purpose of the present article, we consider these two indistinguishable as either would give rise to observed orientation independence.
} 
street aligned with the grid of a city than when traveling a misaligned street. Similarly, Werner and Schmidt found that alignment with strong environmental structure appears to facilitate retrieval. Overall, these foundational observations have established that the accuracy of spatial recall depends on the participant's actual/imagined position when they make judgments about the space.

These various forms of retrieval facilitation might reflect preferred orientations, but they are also consistent with other potential organizations for spatial memory, and the methods were not optimized to distinguish between these possibilities. For example, one possible alternative is that accuracy of spatial memory depends primarily on the familiarity of the specific imagined position. This account would make very different predictions about spatial recall than those of a single preferred orientation that was selected on the basis of familiarity; however, the benefits seen in previous research could be consistent with such a story, since positions aligned with local environmental structure are likely to be more familiar by design (e.g., Lynch, 1960). In addition, these theoretical differences stress the importance of distinguishing between benefits for a particular orientation of the space and benefits for particular positions within the space. A reference orientation should have a global influence over retrieval of any location within a space-even in areas in which the cues that led to its selection are not strong or present. If the direction of a major road drives the selection of a preferred orientation for a city, then recall at that orientation should be more accurate even in a nearby park where the road is not visible.

The present study was designed to directly test whether memory representations of familiar spaces learned via normal daily experience reveal the same orientation dependence observed under the more controlled laboratory learning conditions. To address this question, one must have an environment that has clear boundaries, covers a limited enough area to be a coherent space, and includes locations that can be used to interrogate the accuracy of retrieval from many different orientations. In addition, one must have a captive population that uses said environment for daily navigation. Fortunately, college campuses afford these conditions. A campus is a defined set of buildings, quads, and so on, and students re-experience the same campus environment on a daily basis, in a variety of different contexts (classes, meetings, meals, social activities). Finally, their memories for campus have been built up over timescales of months to years. With these constraints in mind, we used undergraduate students on the Homewood campus of Johns Hopkins University, and asked them to retrieve information about commonly known campus locations from memory.

\section{Method}

Participants

Fifty-eight students (29 female) at the Johns Hopkins University participated in return for extra credit in psychology courses.

Materials and design

Judgments of relative direction Memory was tested with judgments of relative direction (JRDs), in which participants point to targets from imagined headings in space (e.g., Roskos-Ewoldsen et al., 1998; Shelton \& McNamara, 1997). To form these JRDs, we needed to identify buildings on the Homewood campus that would be familiar to the diverse population enrolled in psychology courses. Extensive pilot work identified 17 buildings that were consistently familiar to students, 13 of which fell into the three major quads that form the academic and residential core of the campus. These 13 buildings were combined into pairs defining imagined headings (Fig. 1), and all 13 also served as targets for JRDs. The remaining four buildings were well known but peripheral to the cluster of core buildings, either embedded in the neighborhood across North Charles Street or at peripheral campus locations. These four buildings were never used as part of an imagined heading, but were included as targets for JRDs. These targets afforded an analysis of pointing within, between, and beyond quads, but no differences were subsequently revealed; this issue of target location is not discussed further.

Using the veridical orientations of all possible buildingpair headings, calculated from Google Earth coordinates, we selected those that were roughly aligned with one of eight orientations around the campus $\left(0^{\circ}-315^{\circ}\right.$ in $45^{\circ}$ increments). Unlike laboratory displays, the Homewood campus was not designed to provide exact headings at fixed intervals, so we adopted a $+/-11.25^{\circ}$ tolerance for assigning orientation (e.g., a $7^{\circ}$ heading would be classified as a $0^{\circ}$ heading). We also considered the possibility that the Homewood campus might contain spatial hierarchies, which could influence recall of campus locations (e.g., Hirtle \& Jonides, 1985; Friedman, 2009; Friedman \& Montello, 2006), so we selected building pairs so that half of the pairs at each orientation were buildings located within the same quad (within-quad headings), and half of the pairs were buildings from two different quads (across-quad headings). For each buildingpair heading, all remaining buildings were possible targets. To avoid implicit biases based on our own knowledge and use of the Homewood campus, we randomly selected 16 test trials at each heading type (two) by orientation (eight), yielding 256 trials. 
Fig. 1 A map of the Johns Hopkins Homewood campus, using a West-as-up convention common at Hopkins. Buildings used as stimuli in this experiment are outlined in dark pen. The gray arrows indicate the assignment of orientations. Image courtesy of Johns Hopkins University Office of Undergraduate Admissions

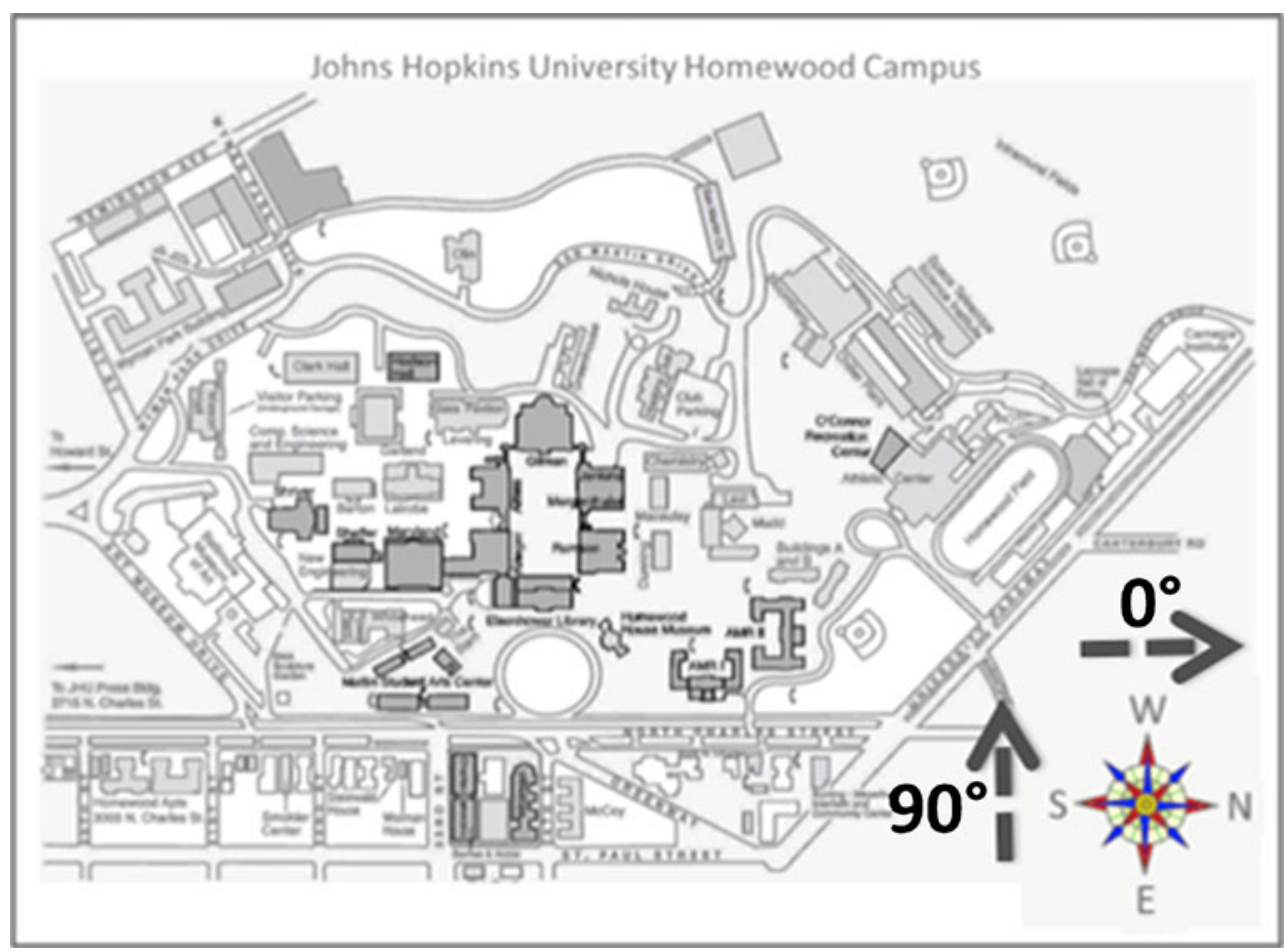

Computerized trials were presented with a pointing wheel consisting of a black circle and a pointer line that originated in the center of the circle and tracked the mouse radially. The name of the building at the to-be-imagined location appeared in the center of the circle (e.g., Ames Hall), and the name of the to-be-faced building appeared at the top of the circle (e.g., Krieger Hall). The name of the target building appeared in a box at the top of the screen (e.g., library), and the participant had up to $15 \mathrm{~s}$ to move the pointer line to indicate the direction to the target object from that heading. If the participant responded before the deadline, the trial ended and pointing judgment direction and response latency were recorded. If participants exceeded $15 \mathrm{~s}$, the computer emitted an aversive beep and recorded a miss. Missed trials were not repeated. There was a $1.2 \mathrm{~s}$ intertrial interval, and participants received a $12 \mathrm{~s}$ rest every 16 trials.

\section{Procedures}

The use of buildings made imagined locations somewhat ambiguous (e.g., participants could imagine themselves at different points in or around a building). To mitigate this ambiguity, we instructed participants to treat each building as a single point (e.g., the center of the building), and to always use this point when imagining or locating the building. Participants were trained to perform JRDs with 16 practice trials using imagined headings that did not correspond to the eight orientations of interest. Following practice, participants were given an opportunity to ask questions before the test trials began. The 256 test trials were then presented in random order.

Campus experience questionnaire Once participants completed the JRDs, they filled out a Campus Experience Questionnaire (CEQ). The CEQ had two components: (a) assessing the participants' familiarity and experience with the Homewood campus, and (b) gauging factors that might relate to their everyday use of the campus (e.g., breadth of exploration in the broader community). In the first section of the CEQ, participants indicated their year of study at Johns Hopkins (e.g., fourth year) and listed all of the residence halls or apartment complexes they had lived in during their study. They then used a 5-point scale to rate their familiarity with the buildings on the Homewood campus, including those used in JRDs (from 1[totally unfamiliar] to 5[familiar]), and listed how many classes they had in each building (for academic buildings), along with how many times a week they visited the building in each of their years at Hopkins. The CEQ was included to allow us to confirm a basic knowledge of the campus and acquire information that might predict reference frame selection.

\section{Results}

First, we compared absolute angular error to reaction time at an individual level to determine whether participants were using a speed/accuracy trade-off during performance 
of judgments of relative direction. In general, we did not observe any tendency for participants to tradeoff speed for accuracy (or vice-versa: mean correlation coefficient,0.02); however, two individuals did show evidence of a nonlinear speed/accuracy trade-off (correlation coefficients of -.5 and -.52 ). On closer inspection, it became clear that these participants had a large number of trials completed in less than $1 \mathrm{~s}$, and that these trials tended to be particularly inaccurate. We ran all analyses with and without these two participants and did not observe any differences.

Next, we verified that participants did not time out on trials in a systematic manner that could influence the validity of our results. Average response latency was $7.7 \pm 3.4 \mathrm{~s}$, half of the $15 \mathrm{~s}$ response deadline, suggesting that the deadline did not serve to limit participants' capacity to reason accurately. Participants missed 11 of 256 trials on average; these missed trials appeared uniformly distributed across our factors of interest (orientation of the imagined heading and heading type) and were consistent with the natural consequences of completing massed trials of JRDs (occasional distraction or boredom). In all of the subsequent analyses, we focus on angular error rather than response latency data; however, the patterns in response latency were consistent with the overall story but showed greater variability.

The present dataset relied on the opportunistic use of campus buildings familiar to the average undergraduate at Johns Hopkins; as a result, we could not easily counterbalance pointing direction to the target across all orientations. True pointing direction is known to have an impact on absolute angular error, with pointing to the front being more accurate than to the sides or back (e.g., Shelton \& McNamara, 2001a). To take pointing direction to target into account, we performed all analyses on mean absolute angular error with pointing direction covaried out at the individual level. (Covarying out pointing direction had an impact on certain orientations, as was expected by the unbalanced pointing directions across headings, but the overall result remained the same regardless of whether we removed the covariate.)

We calculated mean absolute angular error for each individual at each orientation by heading type combination. Absolute angular error was submitted to a mixed-model ANOVA with gender and year of study at Hopkins as a between-participants factor and orientation of the imagined heading $\left(0^{\circ}\right.$ to $315^{\circ}$ in $45^{\circ}$ steps) and heading type (within- or across- quad heading) as a within-participants factor. Withinparticipants effects and contrasts were Geisser-Greenhouse corrected for non-sphericity; all contrasts were corrected for multiple comparisons using Scheffe's procedure.

The primary question was whether we could see a preferred orientation in a familiar space learned under more natural conditions. The main effect of orientation was significant, $F(7$, $350)=5.383, p=.001, \eta_{p}{ }^{2}=0.097$, indicating orientation dependence. Given the unconstrained learning, however, it was not clear what orientation was likely to be most accurate. Therefore, we asked whether any of the eight orientations was more accurate than the others, and, if so, whether there was uniform increased error for all remaining orientations. As is shown in Fig. 2, $0^{\circ}$ was more accurate than all other orientations combined, $F(1,57)=41.16, p<.05$. This contrast was not significant for any other orientation, with the exception of $225^{\circ}$, which was less accurate than all other orientations, $F(1,57)=20.81, p<.05$. To rule out the possibility that particularly inaccurate performance at a single orientation could explain the observed benefit for $0^{\circ}$, we repeated the contrast of $0^{\circ}$ versus all other orientations with $225^{\circ}$ excluded, $F(1,57)=35.59, p<.05$.

In addition to the benefit for $0^{\circ}$, there was also a "seesaw" pattern among non- $0^{\circ}$ orientations: those orientations opposite of and orthogonal to $0^{\circ}\left(90^{\circ}, 180^{\circ}\right.$, and $\left.270^{\circ}\right)$ were more accurately retrieved than were the remaining orientations $\left(45^{\circ}, 135^{\circ}, 225^{\circ}, 315^{\circ}\right), F(1,57)=36.737, p<.05$.

The aforementioned pattern has typically been identified with strong environmental structure benefitting certain nonpreferred orientations (e.g., Shelton \& McNamara, 2001a). However, it can also arise from different participants showing different preferred orientations. To allow for this possibility, we inspected the data at the individual level. First, we observed that all participants showed a reasonably distinct preferred orientation, with the nearest second best being opposite of or orthogonal to the best. Most participants showed performance consistent with the $0^{\circ}$ preferred orientation (31 participants). Critically, individual participants still tended to show the see-saw pattern, suggesting that it was not simply an artifact of different preferences.

With respect to hierarchies, we also observed a significant effect of heading type, $F(1,50)=9.898, p=.003, \eta_{p}{ }^{2}=$ 0.165 , with participants pointing more accurately from within-quad imagined headings than across-quad headings

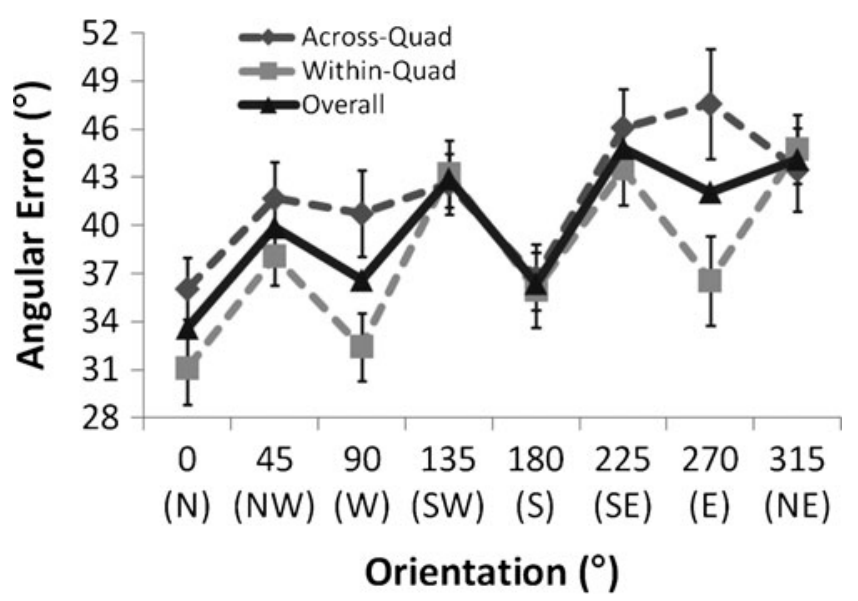

Fig. 2 Average absolute angular error in judgments of relative direction as a function of heading type and orientation of the imagined heading. Error bars reflect \pm 1 SEM 
$\left(38.21^{\circ}\right.$ vs. $\left.41.88^{\circ}\right)$, and a significant orientation $\times$ heading type interaction, $F(7,350)=2.370, p=.038, \eta_{p}{ }^{2}=0.045$. One expectation for this interaction was that the presence or scale of the alignment effect ( $0^{\circ}$ vs. else) could be modulated by the type of heading. Inspection of the data did not support this interpretation; the two heading types showed similar benefits $\left(8.11^{\circ}\right.$ and $6.65^{\circ}$ for within- and across-quad headings, respectively), and the contrast-contrast interaction $\left(0^{\circ}\right.$ vs. else $\times$ within- vs. across-quad $)$ was not significant, $F(1,57)=0.467, p>.05$. Instead, this interaction appears to be due to differential performance on headings orthogonal to the preferred orientation for within- versus across-quad headings, with a stronger benefit for $90^{\circ}$ and $270^{\circ}$ for within-quad versus across-quad headings. This was supported by a contrast-contrast interaction comparing these two advantaged headings to the non-orthogonal headings, $\left(90^{\circ} \& 270^{\circ}\right.$ vs. $45^{\circ}, 135^{\circ}, 225^{\circ}$, and $\left.315^{\circ}\right)$ by across- versus within-quad headings, $F(1,57)=13.372, p<.05$. This could support a difference in the use of the structure available locally (within-quad) versus distally (across-quad), as predicted by hierarchies.

No other effects or interactions were significant. The CEQ provided confirmation of participants' knowledge; however, likely because of the high consistency in the preferred orientation at $0^{\circ}$, we did not observe any factors that appeared to predict principal orientation selection, and none of the effects were sensitive to the number of years that students had been on campus.

\section{Discussion}

We set out to determine whether the orientation dependence of human spatial memory observed in tightly controlled laboratory experiments generalizes to spaces learned under the more natural and highly variable encoding conditions of everyday navigational learning. Our results unambiguously support the prevalence of orientation dependence in spatial memory representations, with our participants organizing campus location along a preferred orientation. This result extends decades of previous research on spatial reference frames from the lab to the real world by showing that similar principles underlie the representations established during a single visit to the lab and those built up through everyday navigation. In addition, this finding has the potential to unite many existing observations about how people recall familiar spaces (e.g., Montello, 1991; Sholl, 1987; Tlauka, 2006; Werner \& Schmidt, 1999) by suggesting that the benefits at recall for alignment with familiar orientations or local structures may reflect the presence of a reference orientation. Our result suggests that these patterns of orientation dependence may reflect a relatively general reference used for all locations within the space, because each of our orientations consisted of trials from multiple different imagined headings. If these previously observed benefits also reflected preferred orientations, then the cues that produced those benefits might also be those that drive selection of a reference for real-world spaces.

The selection of a reference orientation has been a pressing issue in spatial cognition. The consistency in our results was remarkable: Most participants showed a preference for the $0^{\circ} /$ North-as-up orientation. Looking back at Fig. 1, it is clear that the majority of the buildings on campus are located about rectangles that are roughly aligned with this axis. As such, the organizational properties of the participants' memory representations appear to be reflecting obvious physical properties of the structure of the campus (i.e., orientation and alignment of buildings). This apparent use of the structural arrangement of campus as a guiding principle for reference frame selection parallels the findings from a variety of paradigms showing how the organization of objects and object properties can drive the principal reference (e.g., Kelly \& McNamara, 2008; Marchette \& Shelton, 2010; Mou \& McNamara, 2002), as well as the benefit for alignment with environmental structure in recalling familiar spaces (Montello, 1991; Werner \& Schmidt, 1999). Despite the prevalence of North-as-up, we did observe individuals with preferences for other orientations, most often South $\left(180^{\circ}\right)$ or West $\left(90^{\circ}\right)$. An axis facing South might have been selected, since it shares many of the cues that might support a North-facing reference orientation. Similarly, a West reference is orthogonal to this major axis. Moreover, it is consistent with a particular quirk of Johns Hopkins University: Maps of the Homewood campus are typically oriented with West up (although this convention is thankfully changing). Although we did not find a clear case for other idiosyncratic preferences, this variability in the selection of a preferred orientation did raise some additional questions. For example, a small subset of our participants revealed seemingly random preferred orientations that were misaligned with any obvious structural or functional organization of the campus. Although these may reflect properties that we are as of yet unaware, they may also reflect poor selection. We took a cursory look at this intriguing possibility by comparing the overall performance of participants who selected aligned or misaligned orientations. Even at their best orientation, those selecting the aligned orientations performed more accurately than the subset that selected the apparently misaligned orientations. This introduces an interesting question about the role of individual differences in reference frame selection and how this selection relates to success of learning and navigating.

Selection of the reference orientation reflects one potential use of the spatial structure. In addition, we observed elements of a spatial hierarchy influencing memory access. 
Although we had insufficient headings to fully explore preferred orientations in different quads, our data suggest that the selection of North-as-up was not specific to any particular subset of trials. However, we did observe differences in facilitation among non-preferred orientations for within- versus between-quad trials. In particular, orientations orthogonal to $0^{\circ}$ were facilitated more when headings comprised buildings from the same quad, suggesting that within-quad headings allowed participants to make more use of structure to facilitate retrieval. Previous observations of retrieval facilitation (i.e., the see-saw pattern among nonpreferred orientations) have depended on the presence of salient structure. Such a structure may be more readily available when imaging two buildings that are part of the same coherent unit as is the case for buildings that form a quad. (After all, the name "quadrangle" comes from the fact that the buildings tend to form a quadrilateral shape.) Despite the various examples of the see-saw pattern in the literature and now for familiar large-scale space, this retrieval facilitation is an area that is currently underexplored and may represent a rich avenue for future investigations.

Finally, we have attributed the observed preference for North-as-up to the environmental structure because campus buildings are roughly distributed in rectangles with a NorthSouth axis of elongation that is parallel to the major landmark of North Charles Street (Fig. 1). However, there are other features of the environment that might also be driving this selection. First, North-as-up is a map convention with which most, if not all, of our participants are likely familiar. Although it is not the convention for maps at Hopkins, this North-as-up organization may be given priority when learning a new space, because of extensive experience. Second, there is a functional role for the North-South axis on campus: It also serves as the major route of travel for students going from residence halls (as well as much of the off-campus housing) to academic buildings. As such, the reference frame selection could also be driven by salient direction of travel in the space. Data from the CEQ could leverage this aspect, but we had insufficient representation of participants who had extensive experience with primary directions of travel other than the North-South axis: Much of the available housing is on the north end of campus and beyond. Although the coincidence of these various cues on the Homewood campus and our inability to directly manipulate participants' experiences prevent us from disentangling which cues gave rise to the preferred orientation in the present study, this work highlights the need to consider a much broader range of perceptual, conceptual, and experiential cues that might contribute to the formation of spatial memories.

Taken together, these results argue that representing space requires establishing a reference orientation, a requirement that endures over repeated exposures and extensive experience. These results do not suggest that the choice of reference orientation is inflexible with experience. Indeed, it is clear that the preferred orientation can shift with the experience of new viewpoints (Shelton \& McNamara, 2001a) or when updating a representation with new information (Kelly \& McNamara, 2010). The key is that these changes/additions do not mitigate the need to represent space with respect to some orientation. Although our results jibe with well-established principles, the implications may be counterintuitive when we reflect on our own experience of recalling a familiar environment. Subjectively, it feels as though familiar locations from our everyday travels can be easily imagined from many different vantage points; however, it appears that this flexibility does not reflect orientation independence but rather stems from deft manipulation of a representation coded with respect to a single reference orientation.

\section{References}

Acredolo, L. P. (1981). Small- and large-scale spatial concepts in infancy and childhood. In L. Liben, A. Patterson, \& N. Newcombe (Eds.), Spatial representation and behavior across the lifespan (pp. 63-81). New York: Academic.

Anooshian, L. J., \& Young, D. (1981). Developmental changes in cognitive maps of a familiar neighborhood. Child Development, 52, 341-348.

Bryant, K. J. (1982). Personality correlates of sense of direction and geographical orientation. Journal of Personality and Social Psychology, 43, 1318-1324.

Bryant, K. J. (1984). Methodological convergence as an issue within environmental cognition research. Journal of Environmental Psychology, 4, 43-60.

Buttenfield, B. P. (1986). Comparing distortion on sketch maps and MDS configurations. Professional Geographer, 38, 238-246.

Friedman, A. (2009). The role of categories and spatial cuing in global-scale location estimates. Journal of Experimental Psychology. Learning, Memory, and Cognition, 35, 94-112.

Friedman, A., \& Montello, D. R. (2006). Global-scale location and distance estimates: Common representations and strategies in absolute and relative judgments. Journal of Experimental Psychology. Learning, Memory, and Cognition, 32, 333-346.

Greenauer, N., \& Waller, D. (2008). Intrinsic array structure is neither necessary nor sufficient for nonegocentric coding of spatial layouts. Psychonomic Bulletin \& Review, 15, 1015-1021.

Greenauer, N., \& Waller, D. (2010). Micro- and macroreference frames: Specifying the relations between spatial categories in memory. Journal of Experimental Psychology. Learning, Memory, and Cognition, 36, 938-957.

Hintzman, D. L., O’Dell, C. S., \& Arndt, D. R. (1981). Orientation in cognitive maps. Cognitive Psychology, 13, 149-206.

Hirtle, S. C., \& Jonides, J. (1985). Evidence of hierarchies in cognitive maps. Memory \& Cognition, 13, 208-217.

Kelly, J. W., Avraamides, M. N., \& Loomis, J. M. (2007). Sensorimotor alignment effects in the learning environment and in novel environments. Journal of Experimental Psychology. Learning, Memory, and Cognition, 33, 1092-1107.

Kelly, J. W., \& McNamara, T. P. (2008). Spatial memories of virtual environments: How egocentric experience, intrinsic structure, and extrinsic structure interact. Psychonomic Bulletin \& Review, $15,322-327$. 
Kelly, J. W., \& McNamara, T. P. (2010). Reference frames during the acquisition and development of spatial memories. Cognition, 116, 409-420.

Kitchin, R. M., \& Fortheringham, A. S. (1997). Aggregation issues in cognitive mapping. Professional Geographer, 49, 269-280.

Lynch, K. (1960). The image of the city. Cambridge: MIT Press.

Marchette, S. A., \& Shelton, A. L. (2010). Object properties and frame of reference in spatial memory representations. Spatial Cognition and Computation, 10, 1-27.

May, M. (2007). Imaginal repositioning in everyday environments: Effects of testing method and setting. Psychological Research, 71, 277-287.

McNamara, T. P., Rump, B., \& Werner, S. (2003). Egocentric and geocentric frames of reference in memory of large-scale space. Psychonomic Bulletin \& Review, 10, 589-595.

McNamara, T. P., \& Valiquette, C. M. (2004). Remembering where things are. In G. L. Allen (Ed.), Human spatial memory (pp. 324). Mahwah: Erlbaum.

Montello, D. R. (1991). Spatial orientation and the angularity of urban routes: A field study. Environment \& Behavior, 23, 47-69.

Montello, D. R. (1993). Scale and multiple psychologies of space. Lecture Notes in Computer Science, 716, 312-321.

Mou, W., Liu, X., \& McNamara, T. P. (2009). Layout geometry in encoding and retrieval of spatial memory. Journal of Experimental Psychology. Human Perception and Performance, 35, 83-93.

Mou, W., \& McNamara, T. P. (2002). Intrinsic frames of reference in spatial memory. Journal of Experimental Psychology. Learning, Memory, and Cognition, 28, 162-170.

Mou, W., Zhao, M., \& McNamara, T. P. (2007). Layout geometry in the selection of intrinsic frames of reference from multiple viewpoints. Journal of Experimental Psychology. Learning, Memory, and Cognition, 33, 145-154.

Presson, C. C., DeLange, N., \& Hazelrigg, M. D. (1989). Orientation specificity in spatial memory: What makes a path different from a map of the path? Journal of Experimental Psychology. Learning, Memory, and Cognition, 15, 887-897.

Roskos-Ewoldsen, B., McNamara, T. P., Shelton, A. L., \& Carr, W. (1998). Mental representations of large and small spatial layouts are orientation dependent. Journal of Experimental Psychology. Learning, Memory, and Cognition, 24, 215-226.

Rovine, M. J., \& Weisman, G. D. (1989). Sketch-map variables as predictors of way-finding performance. Journal of Environmental Psychology, 9, 217-232.

Shelton, A. L., \& Marchette, S. A. (2010). Where do you think you are? Effects of conceptual current position on spatial memory performance. Journal of Experimental Psychology. Learning, Memory, and Cognition, 36, 686-698.
Shelton, A. L., \& McNamara, T. P. (1997). Multiple views of spatial memory. Psychonomic Bulletin \& Review, 4, 102-106.

Shelton, A. L., \& McNamara, T. P. (2001a). Systems of spatial reference in human memory. Cognitive Psychology, 43, 274-310.

Shelton, A. L., \& McNamara, T. P. (2001b). Visual memories from non-visual experiences. Psychological Science, 12(4), 343-347.

Shelton, A. L., \& McNamara, T. P. (2004a). Orientation and perspective dependence in route and survey learning. Journal of Experimental Psychology. Learning, Memory, and Cognition, 30, $158-170$.

Shelton, A. L., \& McNamara, T. P. (2004b). Spatial memory and perspective taking. Memory \& Cognition, 32, 416-426.

Shelton, A. L., \& Yamamoto, N. (2009). Visual memory, spatial representation, and navigation. In J. R. Brockmole (Ed.), The visual world in memory (pp. 140-177). New York: Taylor \& Francis.

Sholl, M. J. (1987). Cognitive maps as orienting schemata. Journal of Experimental Psychology: Learning, Memory, \& Cognition, 13, 615-628.

Tlauka, M. (2006). Orientation dependent mental representations following real-world navigation. Scandinavian Journal of Psychology, 47, 171-176.

Valiquette, C., \& McNamara, T. P. (2007). Different mental representations for place recognition and goal localization. Psychonomic Bulletin \& Review, 14, 676-680.

Werner, S., \& Schmidt, K. (1999). Environmental reference systems for large-scale spaces. Spatial Cognition and Computation, 1, $447-473$.

Yamamoto, N., \& Shelton, A. L. (2005). Visual and proprioceptive representations in spatial memory. Memory \& Cognition, 33, $140-150$.

Yamamoto, N., \& Shelton, A. L. (2007). Path information effects in visual and proprioceptive spatial learning. Acta Psychologica, $125,346-360$.

Yamamoto, N., \& Shelton, A. L. (2009). Sequential versus simultaneous viewing of an environment: Effects of focal attention to individual object locations on visual spatial learning. Visual Cognition, 17, 457-483.

We are grateful to Andrew Furman for helpful comments on this manuscript, to Joshua Lachewitz and Ram Sundaresh for tireless data collection, and to Robin Sinn for logistical support. This work was partially supported by NSF Cog. Neuro. Grant0920221 to A.L.S. and a Provost Undergraduate Research Award to A.Y. 